\section{AB0612 INFRINGEMENT OF THE BLOOD LIPID SPECTRUM IN CHILDREN AND ADOLESCENTS WITH SLE AND THEIR PREDICTIVE VALUE FOR THE FURTHER COURSE OF THE DISEASE}

N.S. Shevchenko ${ }^{1,2}$, L.F. Bogmat ${ }^{2,3}$, I.M. Bessonova ${ }^{2,3}$, V.V. Nikonova ${ }^{2}$ ${ }^{1}$ department of pediatrics No2, V. N. Karazin Kharkiv National University; ${ }^{2}$ department of cardiorheumatology, Institute of Children and Adolescents Health Care; ${ }^{3}$ department of pediatrics, V. N. Karazin Kharkiv National University, Kharkiv, Ukraine

Background: Infringements of the lipid blood spectrum, or so-called dyslipidemia, are common in adults suffering from SLE and ranges from $36 \%$ to $60 \%$. Many researchers confirmed the accelerated development of atherosclerosis among patients and high risk of epy cardiovascular complications with deterioration of life expectancy. However, the role of prolonged storage of dyslipidemia and subclinical signs of hypercoagulability on the course of the disease is unspecified. Objectives: The aim of the study was to clarify the prognostic importance of the role of storage of metabolic shifts in children with SLE on the background of treatment for the course of the disease.

Methods: A total of 35 people aged 7-18 years with SLE who were ill for more than one year and received complex therapy with glucocorticoids and immunosuppressive drugs were examined. The average age of the patients was 173.56 \pm 4.17 months; the total duration of the disease was $48.45 \pm 3.18$ months. General clinical trials included the complex included autoantibodies, disease activity, drugs. Total cholesterol (TCh), triglycerides (TG), high density lipoprotein cholesterol, low-density lipoprotein cholesterol, apolipoprotein B, ApoA-I and lipoprotein$\alpha$ were evaluated. The state of the blood coagulation system was also studied: fibrinogen of the blood, prothrombin index, thrombin time, active partial thrombin time, d-dimer, international normalised ratio.

Results: The presence of atherogenic dislipoproteinemia in $60.0 \%$ of ill children and adolescents on SLE in children and adolescents, disorders in the system of hemostasis in $25.0 \%$ was established.

The data on the presence of interconnection of blood triglycerides with glomerular filtration rate $(r=-0.892 ; p<0.05)$ and activity of transaminases (alanine transmase) $(r=0.848 ; p<0.05)$

As a result of multiple regression analysis with step-by-step exclusion of minor variables, serum creatinine serum levels in patients with SLE depend on the level of TG of blood with high predictive accuracy: blood creatinine $=0,0672684$ +0.0127034 * $T G ; R=69.79 \% ; R^{2} ;=62.34 \% ; p<0.05$.

A multivariable regression analysis also proved that the level of circulating immune complexes in patients with SFV significantly depends on the level of total cholesterol, complement and TG by the formula: $\mathrm{CIC}=1,44278+0,188976{ }^{*} \mathrm{TCh}-$ 1,51503 * complement $+0,35538$ * $\mathrm{TG} ; \mathrm{R}=94.25 \% ; R^{2} ;=90.02 \% ; p<0.01$.

The mean indices of state of the blood coagulation system indices for children and adolescents with SLE and signs of dyslipidemia were within the norm except for fibrinogen $(2.76 \pm 0.29 \mathrm{~g} / \mathrm{L}$ in the presence of dyslipidemia against $3.72 \pm 0.46 \mathrm{~g} / \mathrm{L}$ in patients with dyslipidemia, $\mathrm{p}<0.05$ ). Its increase persisted in $27.78 \%$ of patients. Conclusions: Thus, violations in the lipid spectrum of blood are often formed in children and adolescents on the background of SLE with the duration of the disease for more than one year. They are not only a risk factor for atherosclerotic lesions; they are also a factor in maintaining the activity of the disease and the development of sustained kidney damage.

\section{REFERENCES:}

[1] Tselios K, Koumaras C, Gladman DD, Urowitz MB. Dyslipidemia in systemic lupus erythematosus: just another comorbidity? 2016 Apr;45(5):60410

Disclosure of Interest: None declared DOI: 10.1136/annrheumdis-2018-eular.7416

\section{$\mathrm{AB} 0613$ \\ CLINICAL AND SEROLOGICAL CHARACTERISTICS OF LUPUS ENTERITIS AND ITS PROGNOSTIC FACTORS IN PATIENTS WITH SYSTEMIC LUPUS ERYTHEMATOSUS}

N. Kajio, Y. Kaneko, R. Anan, J. Kikuchi, T. Takeuchi. Division of Rheumatology, Department of Internal Medicine, Keio University School of Medicine, Tokyo, Japan

Background: Lupus enteritis, known as mesenteric vasculitis including multiple ulcers or ischaemic colitis, and protein-losing enteropathy, is a potentially lifethreatening complication of systemic lupus erythematosus (SLE). Although glucocorticoid therapy is usually effective, some patients experience recurrences or severe complications in that surgical treatment or intensive immunosuppressive therapy is required. Little evidence, however, is available with characteristics and prognostic factors of lupus enteritis.

Objectives: The aim of this study is to clarify clinical characteristics and associated factors for the prognosis with lupus enteritis.
Methods: Consecutive SLE patients in our hospital between 2009 and 2017 were retrospectively reviewed. Patients who developed lupus enteritis which were associated with mesenteric vasculitis or protein losing enteropathy were enrolled. The diagnosis was made by physical examination, contrast enhanced computed tomography and ${ }^{99 m}$ Tc Albumin scintigraphy. Poor prognosis was defined as any surgical treatment or recurrence.

Results: Among 591 SLE patients who fulfilled the 1997 American College of Rheumatology classification criteria, $23(3.9 \%)$ were identified as developing lupus enteritis, and enrolled. Eighteen (78\%) were mesenteric vasculitis and 5 $(22 \%)$ were protein-losing enteropathy. Eighteen $(78 \%)$ were female, the mean age at SLE onset was 31.5 years, and the mean duration between the onset of SLE and lupus enteritis was 10.6 years. The mean SLE disease activity index at the onset of lupus enteritis was 9.6. All patients were treated with glucocorticoid (mean initial predonisolon dose was $40.4 \mathrm{mg} /$ day), and 4 patients $(17 \%)$ were in combination with cyclophosphamide. Among the mesenteric vasculitis patients, 4 patients required surgical treatment and 4 patients experienced recurrence. Comparison between patients with poor prognosis and those without revealed that patients in the poor prognosis group had a higher ratio of multiple ulcer type than ischaemic colitis type ( $43 \%$ vs. $0 \%, p=0.043)$, colon lesions $(71 \%$ vs. $9.1 \%$ $\mathrm{p}=0.012$ ), higher serum $\lg \mathrm{A}$ ( $391 \mathrm{mg} / \mathrm{dl}$ vs. $247 \mathrm{mg} / \mathrm{dl}, \mathrm{p}=0.008$ ), and lower serum total cholesterol (152 mg/dl vs. $191 \mathrm{mg} / \mathrm{dl}, \mathrm{p}=0.012)$. No difference was found in abdominal symptoms, SLE disease activity index score at lupus enteritis onset, anti-dsDNA antibodies and complement levels, initial predonisolone dose, and the concomitant cyclophosphamide use between the two groups. No recurrence was seen in patients with protein-losing enteropathy.

Conclusions: The higher level of serum IgA and lower level of serum total cholesterol are associated with surgical treatment or recurrence and are potential predictive biomarkers for poor prognosis.

\section{REFERENCE:}

[1] Koo BS, et al. Lupus enteritis: clinical characteristics and predictive factors for recurrence. Lupus. 2015; 24: 628-31.

Disclosure of Interest: None declared

DOI: 10.1136/annrheumdis-2018-eular.2749

\section{$\mathrm{AB} 0614$ THE LUPUS STUDIES: THE EUROPEAN AND SPANISH POINT OF VIEW}

N. Bahamontes-Rosa ${ }^{1}$, A. Muñoz Jiménez ${ }^{2}$, F.J. Hidalgo ${ }^{1}$, M.V. Pardo ${ }^{1}$, J.I. Diago Cabezudo ${ }^{1} .{ }^{1}$ GSK, Madrid; ${ }^{2}$ Hospital Virgen del Rocio, Sevilla, Spain

Background: Improving systemic lupus erythematosus patient care comes from the advances performed first in basic science that improved our understanding of the mechanism underlying the pathogenesis and finding effective and safe medications that led to treatment improvement. Furthermore, clinical trials play a key role in advancing both our medical knowledge of the disease and novel treatments.

Objectives: This work aims to offer an overview of the type and characteristics of lupus studies and to analyse the contribution that Europe and Spain have made in supporting those investigations.

Methods: A systematic review of the public registry database of publicly and privately supported clinical studies (CS), ClinicalTrials.gov, was done considering "lupus" as a search criteria (database generated with all CS registered on the 19th September 2017). Data collected included: CT code, title, sponsor, countries par ticipating, study phase, condition and intervention.

Results: Study distribution. By 19th September 2017 there were a total of 256127 CS registered in the world, from which 611 (0.24\%) are associated with lupus studies. From those, $27.2 \%$ are carried out in Europe. Spain is the third country in Europe in number of lupus CS after France and Germany.

Type and distribution of the studies. In the generated clinicaltrials.gov data base the studies are associated to a type of study. The distribution varies depending on the geographical area. In general, the main type of lupus studies conducted in the world are related to the discovery and development of new treatments $(n=406)$ but there are also behavioural, devices investigation, diagnostic test, dietary supplementation, genetic and radiation studies. In Spain, $94.6 \%$ of the studies registered are related to new treatment development.

The distribution of the lupus studies are as follows:

Financial support. $58.6 \%$ of all studies are funded by sources other than industry and $34.8 \%$ are supported by it. In a detailed analysis, $12.5 \%$ of the studies are under the economic support of the National Institutes of Health $(\mathrm{NIH})$ or the partnership of $\mathrm{NIH}+$ other partners. The scenario is different in Europe where industry supported $54.8 \%$ of the studies and in Spain where industry supported $87.3 \%$ of the studies.

Moreover, the funding profile in Spain showed that $92.3 \%$ of the studies associated with new treatments are supported by industry and $7.7 \%$ of them are supported by other sources. On the other hand, $100 \%$ of the studies not related with 